\title{
Compound Bifurcations in the Buckling of a Delaminated Composite Strut
}

\author{
J.E. Wright \\ Centre for Nonlinear Mechanics, \\ University of Bath, Bath, BA2 7AY, UK
}

March 16, 2009

\begin{abstract}
More maths than engineering. Show how simple the model is. Mention the isotropic strut by reference. Focus on the bimaterial strut. Analysis and placement of the bifurcation. Normal form for the bifurcation? Root structure?
\end{abstract}

\section{Introduction}

Using a Rayleigh-Ritz energy formulation we present a simple model for the buckling of the bimaterial strut. The post-buckling results are described in the context of a series of catastrophes with particular parameter combinations resulting in umbilic catastrophes within the double tilted double cusp.

\section{The Simple Model}

\subsection{The geometry of the structure and initial calculation}
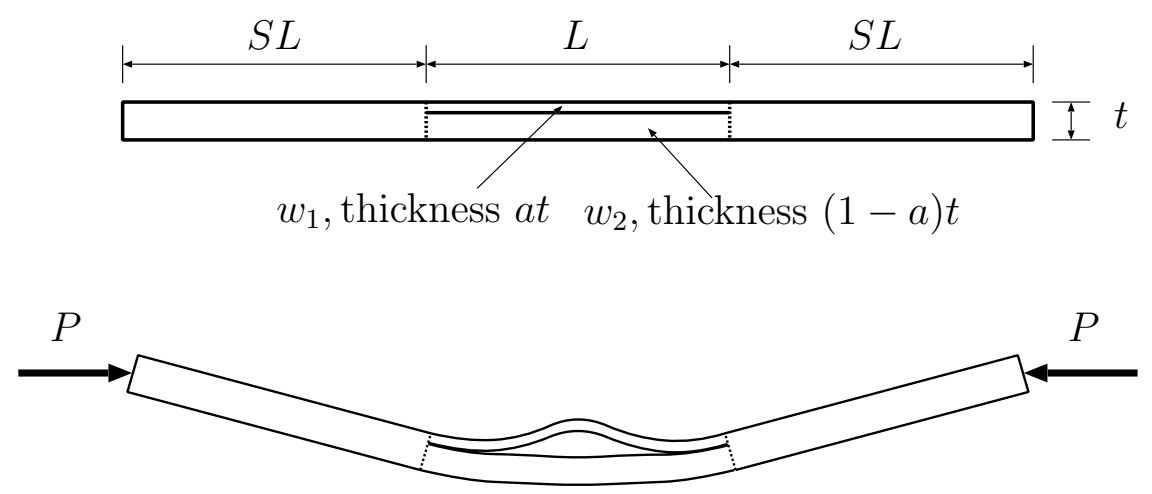

Figure 1: The strut delamination model

The system shown in Figure 1 comprises two undelaminated regions of length $S L$, separated by a region of delamination of length $L$ and subject to a compressive load of 
magnitude $P$. The laminates are two elastic beams of thickness at, $(1-a) t$ and respective buckling displacements $w_{1}, w_{2}$. They are, in effect, struts clamped together at each end by the two undelaminated sections, each of thickness $t$.

Differential stretching between laminates generates nonlinear effects not dissimilar to those seen in plates and shells (1). Separate laminates are therefore given both bending $(E I)$ and in-plane $(E A)$ stiffness, while the undelaminated parts are modelled with bending stiffness alone. The formulation over the delaminated part thus contrasts with a normal strut formulation, for which in-plane stretching can be ignored without losing anything of phenomenological significance (see section ??). Bending stiffness is defined in chapter ?? as a measure of the radius of curvature that will be obtained from a given applied bending moment. It is a measure of the structure's ability to resist deformation and is based on the material properties and geometry of the structure. In this case,

$$
E I=\frac{E t^{3}}{12}
$$

In-plane stiffness is the resistance to stretching and here takes the form

$$
E A=\frac{E t}{2 L}
$$

For the primitive composite strut the layers are attributed different bending stiffnesses, $(E I)_{1}$ and $(E I)_{2}$, and in-plane stiffnesses, $(E A)_{1}$ and $(E A)_{2}$, for the entire length. The undelaminated sections have effective overall bending and in-plane stiffnesses $(E I)_{3}$ and $(E A)_{3}$ respectively. This is achieved by giving the two layers different Young's moduli, $E_{1}$ and $E_{2}$, over the full length of the strut (see Figure 2).

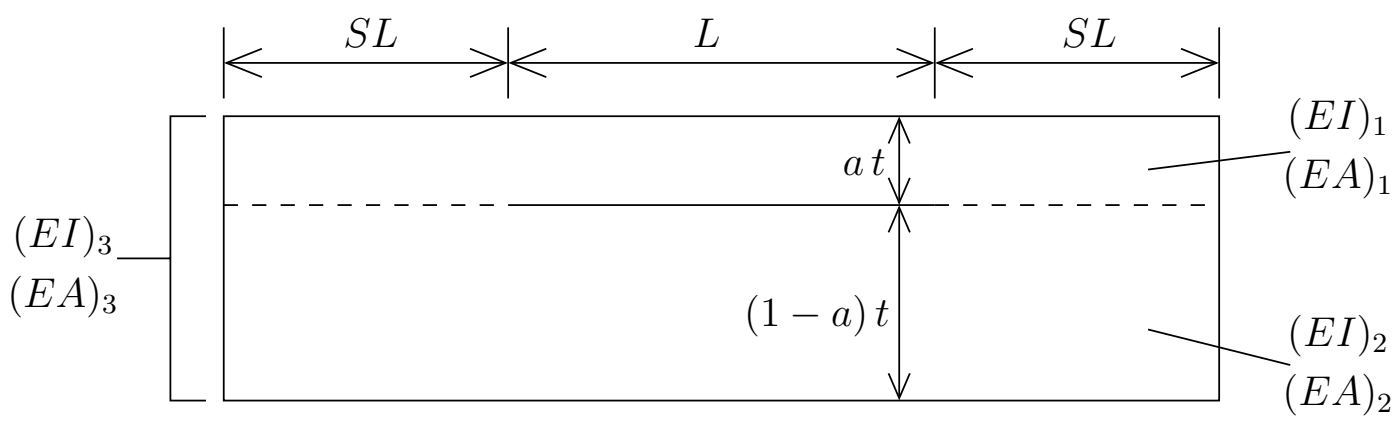

Figure 2: The layout of the bimaterial strut

\subsection{The geometry of the bimaterial strut}

The geometry of the bimaterial strut is similar to that of the isotropic strut. The primary difference is that the neutral axis (NA) no longer coincides with the centre-line (CL). It is offset by the difference in stiffness between the layers.

The position of the neutral axis is defined relative to that of the centre-line, positive if as in Figure 3. Considering moments about the midpoint where moment $=$ force $\times$ distance:

$$
\begin{aligned}
\left(E_{1} a t+E_{2}(1-a) t\right) \gamma & =E_{1} a t \frac{(1-a) t}{2}+E_{2}(1-a) t\left(\frac{t}{2}-\frac{(1-a) t}{2}\right) \\
& =a t \frac{(1-a) t}{2}\left(E_{1}-E_{2}\right)
\end{aligned}
$$




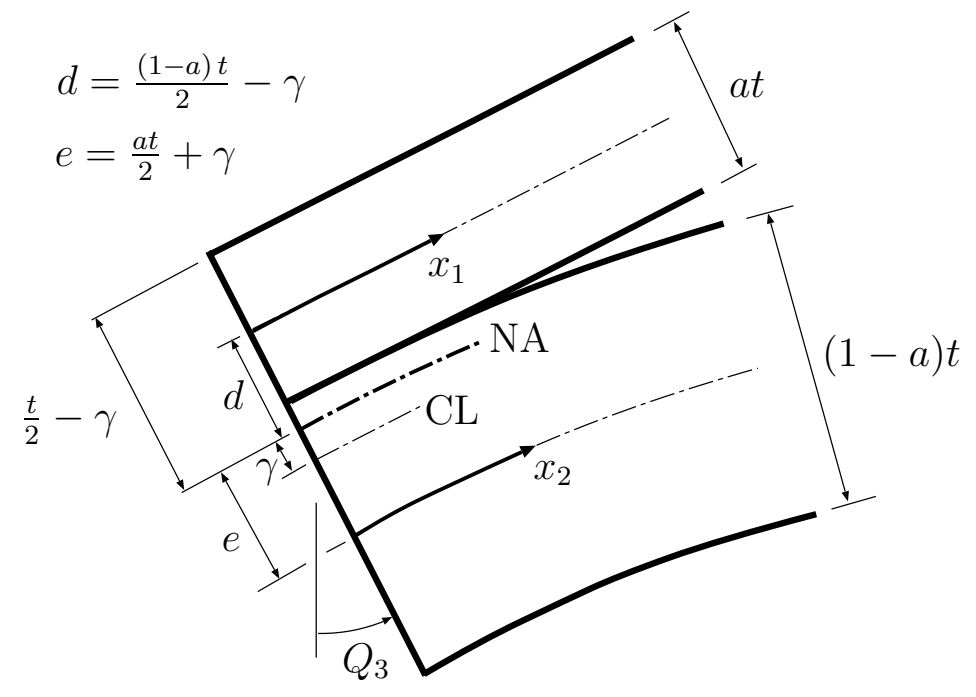

Figure 3: The geometry at the ends of the delaminated region

SO

$$
\gamma=\frac{a(1-a)\left(E_{1}-E_{2}\right) t}{2\left(E_{1} a+E_{2}(1-a)\right)}
$$

The second moments of area about the centre-line of each layer are:

$$
I_{1}=\frac{a^{3} t^{3}}{12}, \quad I_{2}=\frac{(1-a)^{3} t^{3}}{12}
$$

To find the second moment of area of the undelaminated section about the neutral axis the second moments of area of the delaminated sections about the neutral axis are summed. In order to transform the second moments of area above into those required for calculation the Parallel Axis Theorem is used.

Theorem 1 (Parallel Axis Theorem). The second moment of area about an axis parallel to the neutral axis is $I_{A}=I_{N A}+A l^{2}$ where $A$ is the area of the cross-section, $l$ is the distance between the two axes and $I_{N A}$ is the second moment of area about the neutral axis.

To find this the distances $l$ for each laminate are required, shown in Figure 3 as $d$ and $e$ :

$$
\begin{aligned}
d & =\frac{t}{2}-\frac{a t}{2}-\gamma=\frac{(1-a) t}{2}-\gamma \\
e & =\frac{t}{2}+\gamma-\frac{(1-a) t}{2}=\frac{a t}{2}+\gamma
\end{aligned}
$$

where $I_{1}$ and $I_{2}$ are as defined in (4).

Hence, the second moment of area of the undelaminated region about the neutral axis is:

$$
I_{3}=\left[I_{1}+a t\left(\frac{(1-a) t}{2}-\gamma\right)^{2}\right]+\left[I_{2}+(1-a) t\left(\frac{a t}{2}+\gamma\right)^{2}\right]
$$

It is useful to define a parameter $R=E_{1} / E_{2}$, the ratio of the Young's moduli of the layers, that contains all the information about the stiffnesses useful for a parametric investigation. The case of an isotropic strut may be modelled in this way by setting $R=1$. 
This language may also be used to describe a full composite with single delamination. Consider, for example, a strut with two layers above the delamination and ten layers below the delamination. Setting $(E I)_{1}$ to be the overall bending stiffness of the upper two layers, $(E I)_{2}$ to be the overall bending stiffness of the lower ten layers and $(E I)_{3}$ to be the overall stiffness of all twelve layers using second moments of area and the parallel axis theorem would yield a working model of the full composite.

Thus the model is more widely applicable to a general composite with single delamination. Care would be needed as the position of the delamination could no longer be a continuous parameter and would be limited to the positions of the interfaces between layers.

\section{Energy Formulation}

Two significant modelling constraints apply at the ends of the delaminated region. Rotations of the undelaminated region and each laminate must be the same, and there must be no relative shearing movement between laminates at the interface. The first constraint is enforced by the choice of a single degree of freedom, $Q_{3}$, to describe the end rotations, while the second links the amount of axial stretching in each of the laminates to a second degree-of-freedom representing the total end-shortening over the delaminated region, $Q_{4}$.

Both beams obey a coupled system of nonlinear ODEs arising from stationary values of a potential energy function. Rather than solving these, a Rayleigh-Ritz approach is adopted in which suitable mode-shapes for each component are considered and stationary values of the displacement over this restricted class are found. This approach can be highly accurate with judicious choice of mode-shapes $(2 ; 3)$. Evidence of the accuracy of this method for this system is seen in the comparison of the results with finite element results (section ??).

The Rayleigh-Ritz formulation is generated in much the same way as before, the differences arising from the two stiffnesses and the overall stiffness. The approach is the same; with a little additional calculation required.

The same modelling constraints are applied as for the isotropic strut; one is applied by choosing a single degree of freedom, $Q_{3}$, to describe the end rotations, while the second ties the amount of axial stretching in each of the laminates to a second degree-of-freedom representing the total end-shortening over the delaminated region, $Q_{4}$. The buckling displacement of each laminate, represented by its clamped-end buckling mode, supplies two further degrees of freedom, $Q_{1}$ and $Q_{2}$, and provides a Rayleigh-Ritz model similar to that of chapter ??. With $x_{1}$ and $x_{2}$ measuring along the centre line of each laminate as shown in Figure ??, lateral displacements of the laminates can be described by

$$
w_{i}=Q_{i} \sin ^{2} \frac{\pi x_{i}}{L}+Q_{3} \frac{1}{L} x_{i}\left(L-x_{i}\right)
$$

$(i=1$ or 2$)$ for the delaminated parts, and

$$
w_{3}=Q_{3} \frac{\sin \beta x_{3}}{\beta \cos \beta S L}, \text { where } \beta=\sqrt{\frac{P}{(E I)_{3}}},
$$

for the undelaminated parts, $x_{3}$ being measured from the pinned supports along the neutral axis. $(E I)_{3}$ denotes the overall bending stiffness of the undelaminated region. The modeshapes are derived as shown in chapter ??. 
The first term for $i=1,2$ describes the general deflected shape of a strut buckling under axial compression with clamped ends with $Q_{1}$ and $Q_{2}$ being the respective amplitudes of the buckles of each layer (see section ??). The second ensures the correct rotation at the interface and throughout the length by incorporating the effects of shear and bending at the ends (see Figure ??). The shape for $w_{3}$ is chosen to match both the deflection and the end rotation condition at $x_{3}=S L$, in a portion of an equivalent, simply-supported, undelaminated strut. $\beta$ is a coefficient in the governing differential equations of the strut as described in (author?) (4, $k$ in pgs 1-11).

For the case of the primitive composite it is necessary to obtain the bending stiffnesses for each region. Using equations (4) and (5),

$$
\left.\begin{array}{rl}
(E I)_{i} & =E_{i} I_{i} \quad i=1,2 \\
(E I)_{3} & =E_{1}\left[I_{1}+a t\left(\frac{(1-a) t}{2}-\gamma\right)^{2}\right]+E_{2}\left[I_{2}+(1-a) t\left(\frac{a t}{2}+\gamma\right)^{2}\right]
\end{array}\right\}
$$

where

$$
\gamma=\frac{a(1-a)\left(E_{1}-E_{2}\right) t}{2\left(E_{1} a+E_{2}(1-a)\right)}
$$

defines the position of the neutral axis relative to that of the centre line, positive if as in Figure 3 .

\subsection{The potential energy function}

The delaminated strut is modelled here by a four degree-of-freedom potential function, $V\left(Q_{i}\right)$, with a number of parameters. The potential function comprises the difference between energy stored and work done by external loads. The derivation in the case of an undelaminated strut is discussed in more detail in chapter 2. In this system energy is stored in the system through strain energy of bending and stretching.

Under the assumption that each of the layers is in a state of simple bending about its own neutral axis, the first-order strain energy of bending is calculated using equation (??).

$$
\begin{aligned}
U_{B}=\frac{1}{2}(E I)_{1} \int_{0}^{L}\left(\frac{\mathrm{d}^{2} w_{1}}{\mathrm{~d} x_{1}^{2}}\right)^{2} \mathrm{~d} x_{1} & +\frac{1}{2}(E I)_{2} \int_{0}^{L}\left(\frac{\mathrm{d}^{2} w_{2}}{\mathrm{~d} x_{2}^{2}}\right)^{2} \mathrm{~d} x_{2} \\
& +(E I)_{3} \int_{0}^{S L}\left(\frac{\mathrm{d}^{2} w_{3}}{\mathrm{~d} x_{3}^{2}}\right)^{2} \mathrm{~d} x_{3}
\end{aligned}
$$

Similarly, the total axial shortening in each of the laminates can be written as

$$
\begin{aligned}
\delta_{1} & =Q_{4}-\frac{1}{2} \int_{0}^{L}\left(\frac{\mathrm{d} w_{1}}{\mathrm{~d} x_{1}}\right)^{2} \mathrm{~d} x_{1}-((1-a) t-2 \gamma) Q_{3}, \\
\delta_{2} & =Q_{4}-\frac{1}{2} \int_{0}^{L}\left(\frac{\mathrm{d} w_{2}}{\mathrm{~d} x_{2}}\right)^{2} \mathrm{~d} x_{2}+(a t+2 \gamma) Q_{3} .
\end{aligned}
$$

this time incorporating the change in distance due to $\gamma$. With the assumption that the corresponding strains are evenly distributed along the length $L$, the total stretching energy of the system is

$$
U_{S}=\frac{t}{2 L}\left[E_{1} a \delta_{1}^{2}+E_{2}(1-a) \delta_{2}^{2}\right]
$$


This is the source of all the nonlinearities governing post-buckling effects.

A final contribution to the total potential energy comes from the work done by the load $P$ moving through its corresponding deflection $\mathcal{E}$, thus

$$
V=U_{B}+U_{S}-P \mathcal{E}
$$

where

$$
\mathcal{E}=Q_{4}+\int_{0}^{S L}\left(\frac{\mathrm{d} w_{3}}{\mathrm{~d} x_{3}}\right)^{2} \mathrm{~d} x_{3}
$$

the final term describing the end-shortening of the two undelaminated regions. After substituting the deflected forms (6) and (7) into the above, a four degree-of-freedom potential function $V\left(Q_{1}, Q_{2}, Q_{3}, Q_{4} ; P\right)$ is obtained. The solution is found when the gradient of $V$ with respect to $\boldsymbol{Q}$ vanishes, $\nabla_{\boldsymbol{Q}} V \equiv 0$.

This potential function expressed in terms of $Q_{1}, Q_{2}, Q_{3}, Q_{4}$ is a fourth degree polynomial and is given in full in appendix ??. $E_{1}$ and $E_{2}$ both play a part in every term of the equations so varying these should have a significant effect on the system.

\section{Initial Bifurcation: $a<0.5$}

At the fundamental equilibrium solution there is a linear relation between the applied load and axial displacement. The critical load at which initial buckling from the fundamental solution occurs is again characterized by transcritical bifurcation.

As discussed in chapter ??, the critical load occurs when $\left|V_{i j}^{C}\right|=0$, i.e. when $|H(V)|=$ $\operatorname{det}(H(V))$, where $H$ denotes the Hessian and superscript $C$ indicates evaluation at the critical point. Equivalently, it is required that one or more eigenvalue of $H(V)$ satisfies $\lambda_{i}=0$. As with chapter ??, the first step is to reduce the potential function to depend on precisely three degrees-of-freedom and measured from the fundamental path. Reduction of the potential function is carried out first in the same way as for chapter ?? and $V$ is reduced to a function of the form $V\left(Q_{i} ; \Lambda^{j}\right),(i=1,2,3)$ which has three degrees-of-freedom. The quadratic terms of energy can be represented by Taylor coefficients, related to expansion about the fundamental equilibrium path where $Q_{i}=0, i=1,2,3$

$$
\begin{aligned}
V_{11}^{F} & =\frac{1}{6} E_{1} a^{3} t^{3} L\left(\frac{\pi}{L}\right)^{4}-\frac{1}{2} K_{1} P a L\left(\frac{\pi}{L}\right)^{2} \\
V_{22}^{F} & =\frac{1}{6} E_{2}(1-a)^{3} t^{3} L\left(\frac{\pi}{L}\right)^{4}-\frac{1}{2} K_{2} P(1-a) L\left(\frac{\pi}{L}\right)^{2} \\
V_{33}^{F} & =\frac{t^{3}}{3 L K_{3}}-\frac{1}{3} \sqrt{\frac{P}{K_{3}}}\left[\sqrt{3} \tan \left(2 \sqrt{3 P K_{3}} S L\right)+\sqrt{P K_{3}} L\right] \\
V_{12}^{F} & =0 \\
V_{13}^{F} & =-P a K_{1} \\
V_{23}^{F} & =-P(1-a) K_{2} .
\end{aligned}
$$

where the $K_{i}$ are defined as follows:

$$
\begin{aligned}
K_{1} & =\frac{E_{1}}{E_{1} a+E_{2}(1-a)} \\
K_{2} & =\frac{E_{2}}{E_{1} a+E_{2}(1-a)} \\
K_{3} & =\frac{E_{1} a+E_{2}(1-a)}{E_{1}\left(E_{1}-E_{2}\right) a^{4}-E_{2}\left(E_{1}-E_{2}\right)(1-a)^{4}+E_{1} E_{2}}
\end{aligned}
$$


Here subscripts of $V$ denote partial differentiation with respect to the corresponding degreeof-freedom (5). Corresponding critical mode-shapes can then be obtained directly from the associated eigenvectors (6):

$$
\left.\frac{Q_{1}}{Q_{3}}\right|^{C}=-\left.\frac{V_{13}}{V_{11}}\right|^{C} ;\left.\quad \frac{Q_{2}}{Q_{3}}\right|^{C}=-\left.\frac{V_{23}}{V_{22}}\right|^{C}
$$

onestiffcrit.ps not found

(a)

\section{bimatcrit.ps not found}

(b)

Figure 4: The variation of the critical load with delamination depth for $S=0.5$ and (a) $R=E_{1} / E_{2}=1$, (b) $R=E_{1} / E_{2}=10$

mode1new.ps not found

(i) mode2.ps not found

(ii)

Figure 5: Mode-shapes at critical loads for $a=0.00001$ and $S=0.5$. (i) Lower critical load (closing) and (ii) higher critical load (opening)

In Figure 4 the two lowest critical loads are shown as functions of the delamination depth parameter, $a$, normalized by the Euler load

$$
P_{E}=\frac{\pi^{2}(E I)_{3}}{(L(1+2 S))^{2}}
$$

of a corresponding undelaminated strut of length $L+2 S L$ for (a) an isotropic strut and (b) a two layer composite strut.

The mode-shapes for the delaminated region depicted in Figure 5 are referred to as the "closing" and "opening" modes respectively. As for the isotropic strut, the lowest critical load always gives rise to the closing mode; however, the opening mode is seen to play a significant role in the post-buckling regime (see section ??).

A change is seen to take place near $a=0.25$, the critical delamination depth, similarly to the isotropic strut. If $a$ is less than this value the response is largely dominated by buckling of the thinner laminate; if $a$ is greater and response is described by the flatter central region, the Euler load is almost reached and the delamination is apparently less important. This is also the same in the region of $a=0.79$. When $a$ becomes greater than this value the buckling of the thinner laminate dominates again.

\subsection{The double eigenvalue}

Figure 4(b) shows the two critical loads becoming very close near $a=0.79$. This interaction is key to understanding the complex secondary bifurcations observed in the post-buckling response of the bimaterial strut, and, in particular, the bifurcations central to the umbilics in this structure shown in Figure 11.

The point at which the double eigenvalue occurs, a point in parameter space, will, if it exists, provide the central "umbilic point" at which all the paths come together. Both primary bifurcations at the critical points and both secondary bifurcations coincide at this point. It is postulated that this point will compose of $\left(Q_{1}, Q_{2}, Q_{3}, Q_{4} ; P, a, S, R, \varepsilon\right)$ precisely, where $\varepsilon$ is an imperfection parameter. 


\subsection{The Numerical Methods for the Post-Buckling Regime}

\subsection{Problem}

The post-buckling response is analysed by determining paths of equilibrium states in the applied load and end-shortening plane. Equilibrium occurs at points $\left(Q_{1}, Q_{2}, Q_{3}, Q_{4} ; P, E, t, a, L, S\right)$ for which

$$
\frac{\partial V}{\partial Q_{i}}=0, \quad i=1,2,3,4 .
$$

NOTE THAT THE PARAMETER $R=E_{1} / E_{2}$ is included and eqbm has $\left(Q_{1}, Q_{2}, Q_{3}, Q_{4} ; P, E_{2}, R, t, a, L\right.$ Also add in the following modifications to the method: $E_{1}$ is wholly dependent on $R$ and $E_{2}$ so is not important here; instead it is calculated while running the code as $E_{1}=R \times E_{2}$. The useful and informative parameter is $R$ as this contains the possibilities of $E_{1}$ and $E_{2}$ concisely. The input parameters are now $\left(E_{2}, R, t, a, L, S\right)$. Various formulae had to be altered in the code used for the isotropic strut and it was necessary to calculate the second moment of area for the undelaminated section at each step in the homotopy code where $a$ is a variable. This was used in the calculation of the total end-shortening at each point. The main modifications arose in the Maple code used to find the potential function. This then supplied most of the changes necessary to the Fortran code by means of an inbuilt translator in Maple.

Optimization schemes are generally directed towards finding minima of an objective function, the maxima and saddles usually being of less interest. In the case of controlled end-shortening, however, equilibrium states that are saddle points are also likely to be stable. The equilibrium states are described diagrammatically in section ??.

The problem is formally defined in the following terms:

Definition 1 (Problem). Find the minima and saddles of a general potential function $V\left(Q_{i} ; \Lambda^{j}\right)$ for varying $\Lambda^{1}$ for a number of sets of parameters for $\left(\Lambda^{2}, \ldots, \Lambda^{m}\right)$.

The method detailed here uses a variety of mathematical techniques, notably scaling and homotopy, to efficiently and reliably determine the minima, saddles and maxima of a potential function describing a system that varies on two distinct displacement-scales.

The underlying mathematics is presented first. Then the method is discussed within the context of the delaminated strut problem and the required mathematical techniques are introduced.

\subsection{Numerical method}

Traditionally, solvers of nonlinear systems, often based on Newton-Raphson methods, search for minima only. Lemma 4.1 provides a means of using such a solver to determine saddles and maxima as well.

Lemma 4.1. Let $V: \mathbb{R}^{n} \rightarrow \mathbb{R}$ and choose $F: \mathbb{R}^{n} \rightarrow \mathbb{R}$ such that

$$
F=\frac{|\nabla V|^{2}}{2}
$$

If $\boldsymbol{Q}$ is a point in $\mathbb{R}^{n}$ and $\nabla V(\boldsymbol{Q})=0$ then $\nabla F(\boldsymbol{Q})=0$ and at such a point

$$
H(F)=H(V)^{2}
$$

where $H$ denotes the Hessian. 
Proof. Let $V: \mathbb{R}^{n} \rightarrow \mathbb{R}$ and $F: \mathbb{R}^{n} \rightarrow \mathbb{R}$ such that

$$
F=\frac{1}{2} \sum_{i=1}^{n}\left(\frac{\partial V}{\partial x_{i}}\right)^{2}
$$

Then

$$
\frac{\partial F}{\partial x_{j}}=\sum \frac{\partial V}{\partial x_{i}} \frac{\partial^{2} V}{\partial x_{i} \partial x_{j}}
$$

Hence,

$$
\nabla F=H(V) \nabla V
$$

where the Hessian is defined as $H_{i j}=\partial^{2} V / \partial x_{i} \partial x_{j}$. Therefore, if $\nabla V=0, \nabla F=0$.

Differentiating equation (16) yields

$$
\frac{\partial^{2} F}{\partial x_{j} \partial x_{k}}=\sum \frac{\partial^{3} V}{\partial x_{j} \partial x_{i} \partial x_{k}} \frac{\partial V}{\partial x_{i}}+\sum \frac{\partial^{2} V}{\partial x_{j} \partial x_{i}} \frac{\partial^{2} V}{\partial x_{i} \partial x_{k}}
$$

At a turning point, the product

$$
\left(\frac{\partial^{3} V}{\partial x_{j} \partial x_{i} \partial x_{k}}\right)\left(\frac{\partial V}{\partial x_{i}}\right)=0
$$

and thus

$$
H(F)=H(V)^{2}=H^{T}(V) H(V)
$$

As $H(V)$ is symmetric it follows that $\mathrm{H}(\mathrm{F})$ is positive definite.

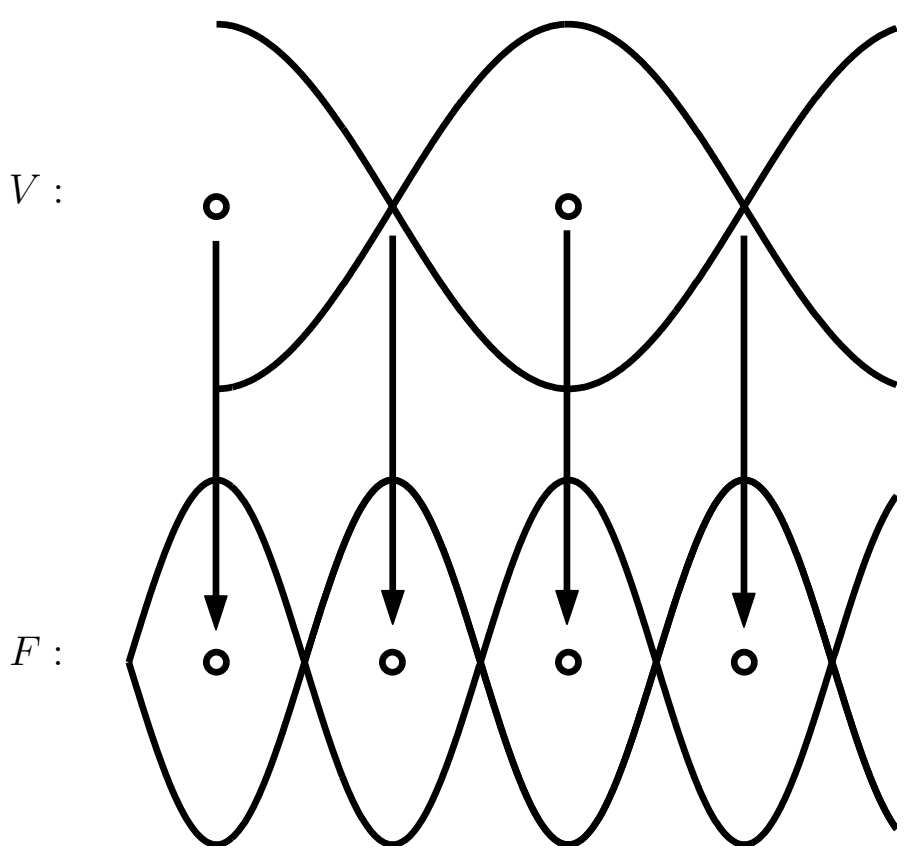

Figure 6: The relationship between stationary points in $V$ and $F$

To find such states numerically a minimum in $F=|\nabla V|^{2} / 2$ is searched for since, by Lemma 4.1, this gives all the required stationary points in $V$; see Figure 6.

Varying a number of $\Lambda^{j}$ simultaneously can yield further physical and mathematical insight but must be implemented with care. The number of variable control parameters 
is led by the number of parameters required to remain at the bifurcation point but this may require the introduction of non-physical parameters. This is considered to produce artificial results so here we restrict our study to the variation of parameters with identifiable physical significance.

For the delaminated strut described the $V$ function to be solved is given in appendix ??. Here, $n=4$ and the $x_{i}$ of the lemma are the $Q_{i}$. There are a number of parameters which may be fixed: $P, E, t, a, L, S$. In the terminology of definition 1 (section 4.3) and (author?) (7), $P$ is chosen to be the special bifurcation parameter, $\Lambda^{1}$, while $(E, t, a, L, S)$ correspond to the remaining $\Lambda^{j}$. Hence, the equations

$$
\frac{\partial V}{\partial Q_{i}}=0, \quad i=1,2,3,4
$$

are solved for fixed $\Lambda^{1}$ using Lemma 4.1 and $\Lambda^{j}$, then $\Lambda^{1}$ is incremented slowly and the equations solved at each step. Thus a path of equilibrium solutions is traced out.

\subsection{Implementation}

The method was implemented using DNSCOMP, a subroutine written in Fortran 77 that solves systems of nonlinear equations within a given tolerance level. It was written by K. L. Hiebert of SNLA and is a modification of the Powell hybrid method (8). The other techniques were implemented for this work directly into a Fortran 77 path-following routine that calls DNSCOMP for each parameter set.

The tolerance level was set to tol $=1.0 \times 10^{-9}$. This means that computation is considered to be sufficiently accurate when the relative error between the estimate of the solution and the actual solution is, at most, tol.

\subsection{Parameters}

The specialized parameter $P$ is varied throughout the calculations so that a path of equilibrium solutions may be traced out relative to it.

Different values of $a$ are selected to provide equilibrium paths for a range of values. This can also be done for $S$; however, the value of $S$ was held constant throughout the analysis unless otherwise stated.

The remaining parameters were held constant at the following values:

$$
\begin{aligned}
E & =70 \mathrm{GPa} \\
t & =2.13 \mathrm{~mm} \\
L & =55 \mathrm{~mm} \\
S & =0.5
\end{aligned}
$$

These values were chosen to correspond with those being used in related experimental and finite element studies being undertaken at the University of Bath at the time (2).

ADD FOLLOWING BIT IN: In order to observe and fully unfold the bifurcational behaviour it is necessary to vary a sufficient number of the correct "type" of parameters ("type" here referring to the effect of the parameter, e.g. tilting, splitting, etc). $P$ is the special bifurcation parameter as in previouschapters. Further parameters chosen to be varied are $a, S, R$. The influence of these parameters will be discussed later. For the purposes of the following analysis $R:=10, E_{2}:=70, t:=2.13, L:=55, S:=0.5$ unless stated otherwise. It is worth noting that the system is still considered to be perfect, that is, there are no geometrical or material imperfections. 


\subsection{Initial guesses and running the code}

The initial guess for the fundamental path is always chosen to be $\left(Q_{1}^{i}, Q_{2}^{i}, Q_{3}^{i}, Q_{4}^{i}\right)=$ $(0,0,0,0.1)$. Since the first three guesses are precise this is sufficient to yield the full path whatever $Q_{4}^{i}$ is guessed to be.

The first initial guess for anything other than the fundamental path is a guess based on where the critical loads are and known possible mode-shapes.

The second initial guess provided to the code is the solution which was calculated for the first value of $P, Q^{1}$. The third and subsequent initial guesses are linear extrapolations based on the solutions for the previous two values of $P, Q^{i-2}, Q^{i-1}$.

The code traces an equilibrium path for 100000 points in less than 1 second and the slowest step appears to be the printing of the solution both to screen and to file. However, it does sometimes fail to solve the system after the solver exceeds its own set options (e.g. maximum number of iterations, maximum number of function evaluations, etc). This can be due to scaling problems (see section 4.9), solution paths being very close together both in magnitude and direction, or the path turning a sharp corner (thus rendering the linear extrapolation an inefficient method for finding the initial guess). These problems can be remedied by re-scaling the computational variables, changing the step size in $P$, changing the initial guess or by re-parameterization.

\subsection{Re-parameterization}

Paths are most obviously obtained by varying our special parameter $\Lambda^{1}=P$ but this is not effective once a limit point in $P$ is reached. It is therefore necessary to parameterize in one or more of the $Q_{i}$ in order to continue the solution path. This is an immediate step and may be achieved simply by controlling the variation in the chosen $Q_{i}$ and making $P$ one of the solution variables. The equations used to define the problem remain the same.

\section{$4.9 \quad$ Re-scaling}

It is quite likely that in many regions of our solution space the rate of change of $\partial V / \partial Q_{i}$ either side of the equilibrium point in question may be small for some $Q_{i}$ and extremely large for others. In this case the numerical problem is said to be ill-conditioned and it is necessary to scale the solution variables during computation both to improve the accuracy of the solution and the efficiency of computation. The initial guesses are multiplied by factors to ensure they are all of the same order of magnitude prior to the solution step; the solution output is then re-scaled back to the physical quantities.

\subsection{Homotopy}

The technique of homotopy can also prove useful for determining the behaviour of the structure under consideration for different values of the $\Lambda^{j}$. Here, small, controlled variations in a chosen parameter, $\Lambda^{2}$ say, allow the use of one equilibrium path corresponding to $\Lambda_{e}^{2}$ to find another equilibrium path for $\Lambda_{f}^{2}$. This is achieved by varying $\Lambda^{2}$ whilst holding $\Lambda^{1}$ and the other $\Lambda^{j}$ constant and then finding the equilibrium solution for $Q_{i}$. Homotopy assists the understanding of how the equilibrium paths change for different sets of parameter values, and gain an understanding of the effect of changes in all the parameters.

Homotopy can be performed over any of the system parameters. It is useful for example to hold $Q_{1}$ constant, then subject $a$ to controlled variation and solve for $\left(P, Q_{2}, Q_{3}, Q_{4}\right)$ as shown in Figure 7. 


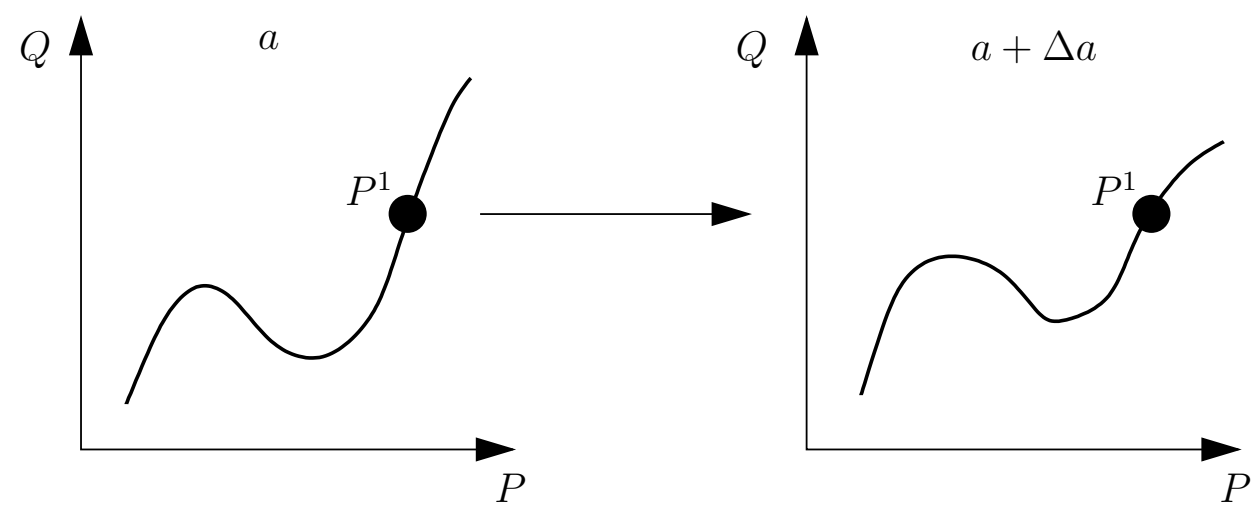

Figure 7: The homotopy process: moving from one equilibrium path to another

\subsection{Verification}

Initial verification was achieved by comparison with results obtained in finite element analyses and Maple (see section ??). The code that was adapted to include re-parameterization, re-scaling and homotopy was verified by quantitative and qualitative comparison with the results of the initial code which used controlled variation in $P$.

ADD IN: The primary method of verification of the code was by setting $R=1.0$ and comparing quantitatively and qualitatively with the results obtained using the method of chapters ?? and ??. For other values of $R$, the code was tested by comparing calculated values with those calculated using the Maple code.

\subsection{Secondary bifurcations: $a<0.5$}

The legend in Table 1 applies to all figures throughout this chapter and descrbes the use of line styles in the plots.

solidblack.ps not foundhe physical line coming from $P_{1}^{C}$ prior to the limit point associated with the secondary bifurcation.

dashblack.ps not foundThe path from $P_{1}^{C}$ beyond the limit point. This path is involved in secondary bifurcations.

dashdotblack.ps not foundhis path joins onto the path from $P_{1}^{C}$ following the secondary bifurcations.

dashgrey.ps not found This path only joins with the path from $P_{1}^{C}$ at the precise point of secondary bifurcation.

dashdotgrey.ps not foundefore the secondary bifurcations this path is the physical path coming from $P_{2}^{C}$.

solidgrey.ps not foundAfter the secondary bifurcations this path is the physical path coming from $P_{2}^{C}$.

crossgrey.ps not foundNon-physical solutions in which the layers have passed through eachother.

Table 1: Legend describing line types used throughout this chapter

The different post-buckling responses for selected delamination depths over the range of $a$ in the composite strut with $R=E_{1} / E_{2}=10$ are shown in normalized load-endshortening plots with inset mode-shapes in Figures 8 and 9. Line types are used consistently 
to indicate the individual paths as in chapter ??. The delamination phenomena described in chapter ?? of thin-film buckling (shown at $a=0.2, a=0.8$ ) and overall buckling (shown at $a=0.25, a=0.75)$ are also observed in the post-buckling of the composite strut. In all four cases the mode-shape marked with a cross at initial bifurcation from the undeflected state has both layers moving in the same direction, with the deflection of the thinner layer being larger. The crossed grey lines indicate unreachable solutions where the layers have passed through one another. The bulleted mode-shapes in Figures 8(a) and 9(b) are what are described as the opening mode of thin-film buckling in which the layers move in opposite directions.

For $a>0.5$, buckling is dominated by $Q_{2}$ as the thinner layer and $Q_{1}$ is the one which undergoes reversal in direction for the opening configuration.

The bulleted mode-shape of Figure 8(b) demonstrates the overall buckling mode. In the case of $a>0.5$, however, contact is achieved for little change in the end-shortening.

Note also from Figure 9(a) that for general buckling, when the thicker layer is the stiffer, the layers contact and pursue Euler buckling as a single undelaminated beam very soon after initial bifurcation.

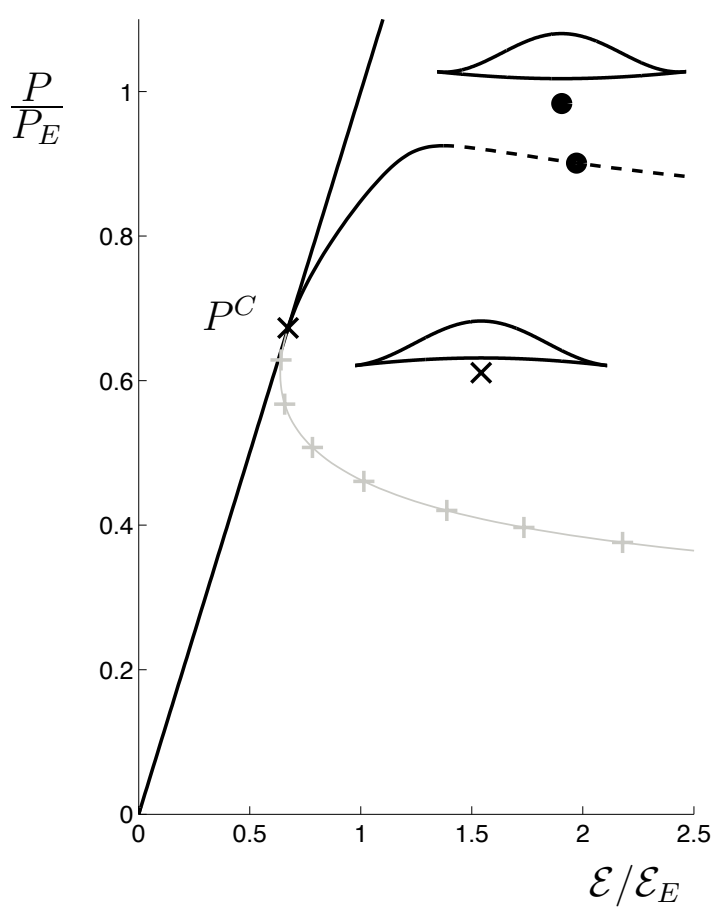

(a)

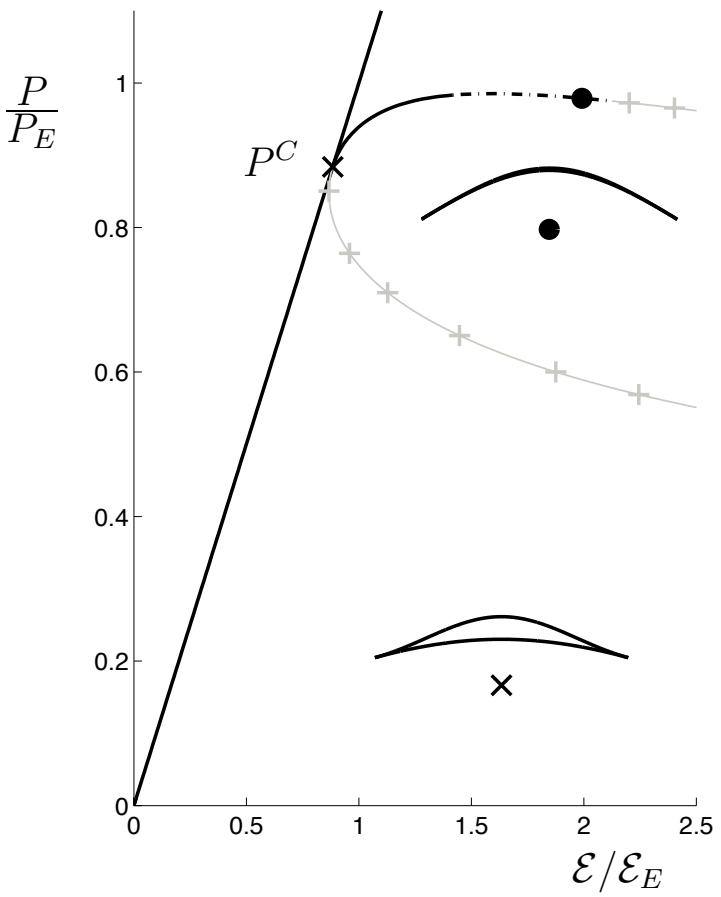

(b)

Figure 8: Post-buckling load - end-shortening plots, (a) $a=0.2$ and (b) $a=0.25, R=$ $E_{1} / E_{2}=10$

Figure 10 shows that the switch from the opening response to the closing response again occurs at a transcritical secondary bifurcation described by $P$ and $a$. The response here, where the thinner layer is formed from the stiffer material, is very similar to that of the isotropic strut of chapter ?? $(2 ; 9)$ except for changes in the response of $Q_{2}$. Unlike the isotropic strut, however, $Q_{2}$ reverses early on for $a=0.2$ so the layers move in the same direction once again but never achieve contact (see Figure 10(c) \& (e)). Comparison of these plots with those for the isotropic strut (Figures ??, ??, ??) shows that these provide considerably more potential for complex response. For example, the loop shown in Figure 10(e) suggests a double reversal response in which the layers first close then open then 


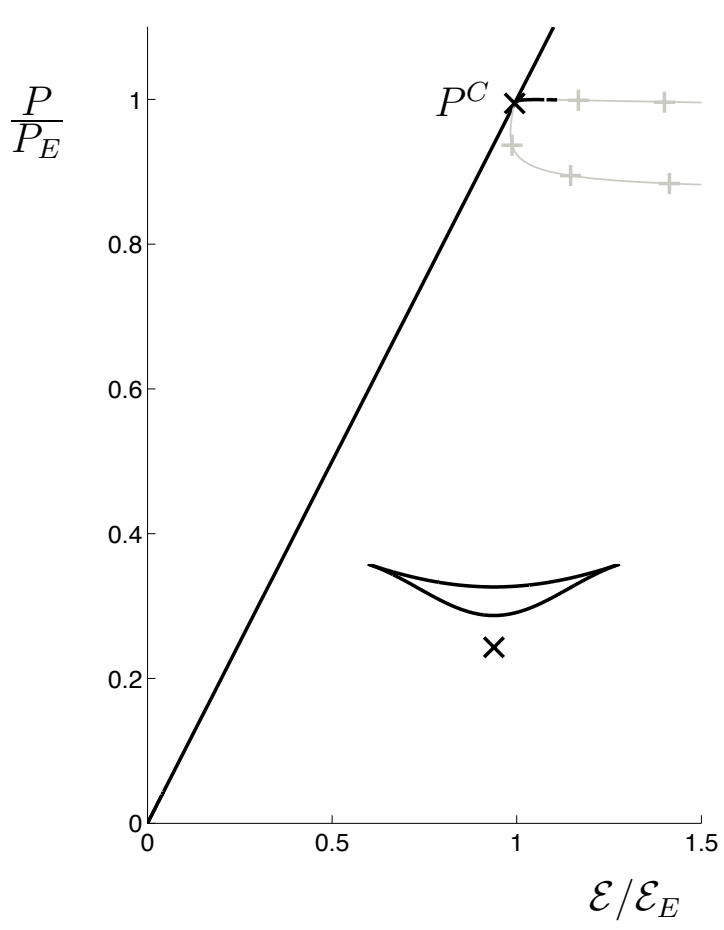

(a)

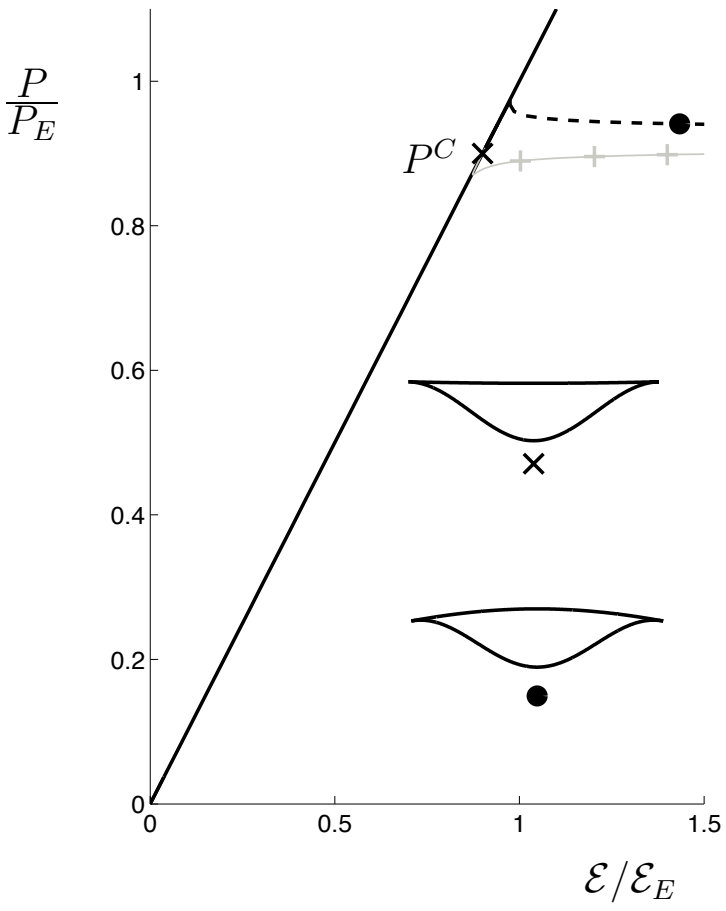

(b)

Figure 9: Post-buckling load - end-shortening plots, (a) $a=0.75$ and (b) $a=0.8, R=$ $E_{1} / E_{2}=10$

close again. The path emerging from the second critical load, $P_{2}^{C}$, plays no part except to effect a tilt in the bifurcation. It is involved in further bifurcations in unstable equilibrium paths at higher load values. These are not examined here as they have no bearing on the physical behaviour of the system and those of $a>0.5$ are of more interest.

The case where the thicker layer is the stiffer is shown in Figure 11. Here, the transcritical secondary bifurcations are again found to be central to the switch in response. The changes are most easily seen in the $Q_{2}$ plots ((e) and (f)) but enlargements of the areas closest to bifurcation are provided as insets in other plots. At $a \approx 0.795$ six paths are found to approach and two points of secondary bifurcation occur very close together and very close to the fundamental path. This makes the path from $P_{2}^{C}$ relevant in defining the physical behaviour of the system and providing another possible route for the postbuckling response. The response becomes highly unstable and unpredictable as there are many possibilities, some of which may be unsafe, particularly if there are imperfections present.

\subsection{Compound Bifurcation: $a>0.5$}

In Figure 11 it appears as though the six paths will all intersect at one point. However, detailed examination shows that this structure is actually two distinct bifurcations occurring very close together. See Figure 13 and the description in section ?? for analysis of how these paths connect at bifurcation.

Varying $S$ either brings the bifurcations closer together in $a$ or moves them further apart, but no value of $S$ can cause them to occur at exactly the same point in $(P, a, S)$ space. So clearly $S$ is an important parameter in unfolding this phenomenon, although it acts in a similar sense to $a$ in chapter ??. $R$ is not classified as an unfolding parameter as the same behaviour will be observed in a neighbourhood of $R=10$. 


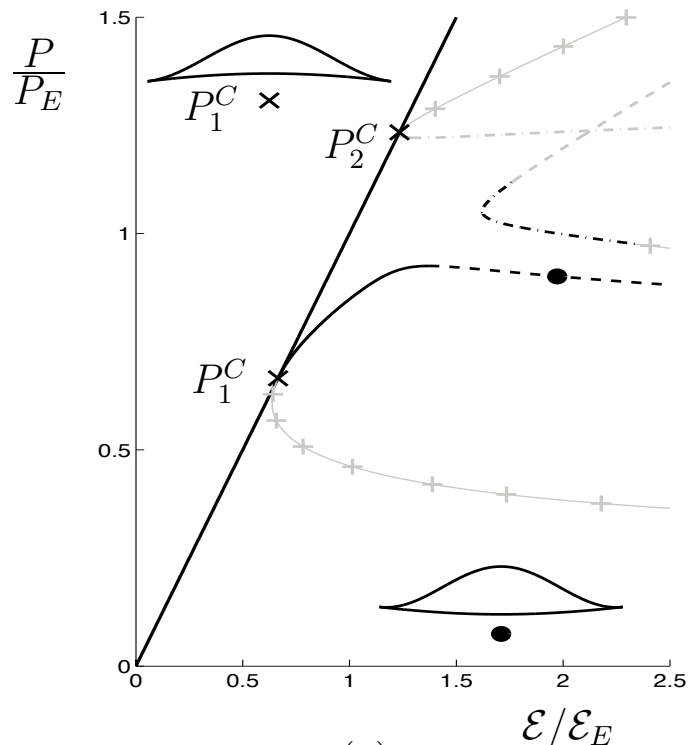

(a)

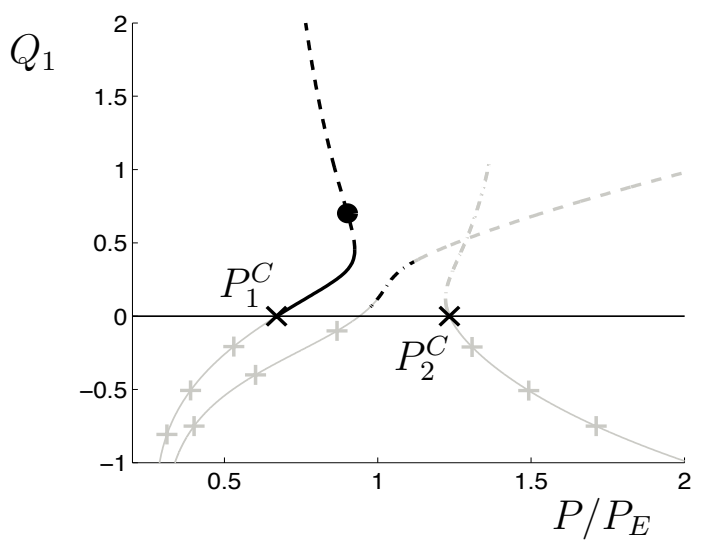

(c)

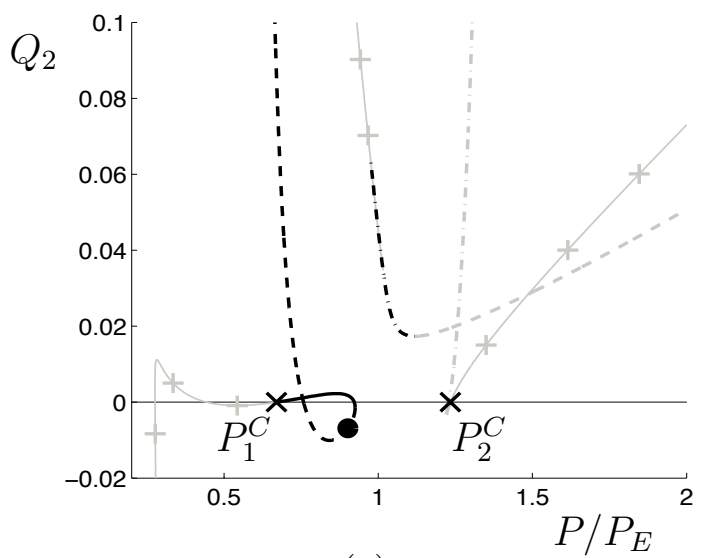

(e)

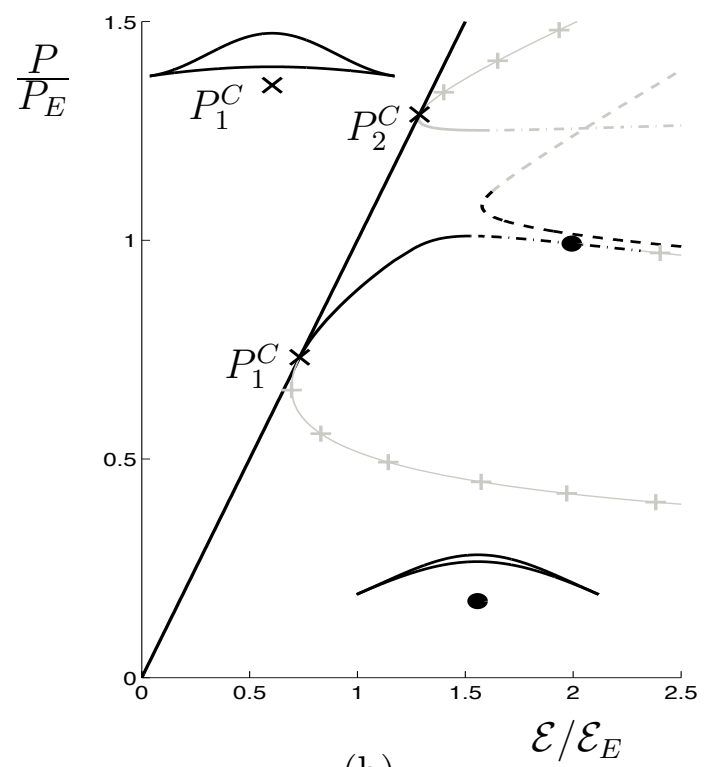

(b)

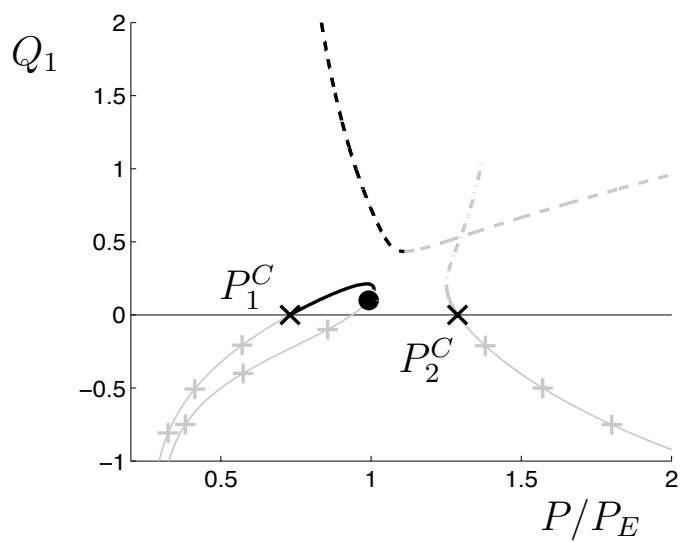

(d)

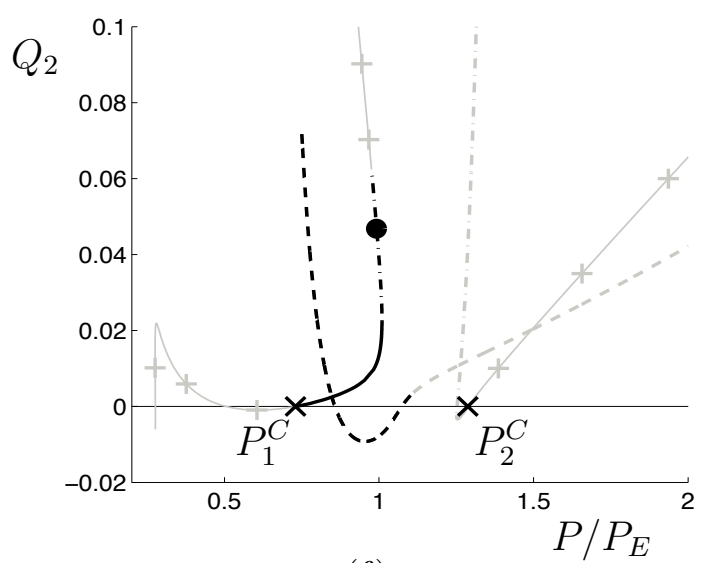

(f)

Figure 10: Load-end-shortening plots for $S=0.5, E_{1} / E_{2}=10$ and (a) $a=0.2$, (b) $a=0.21$. Figures (c) - (f): The variation of the deflection with load. 


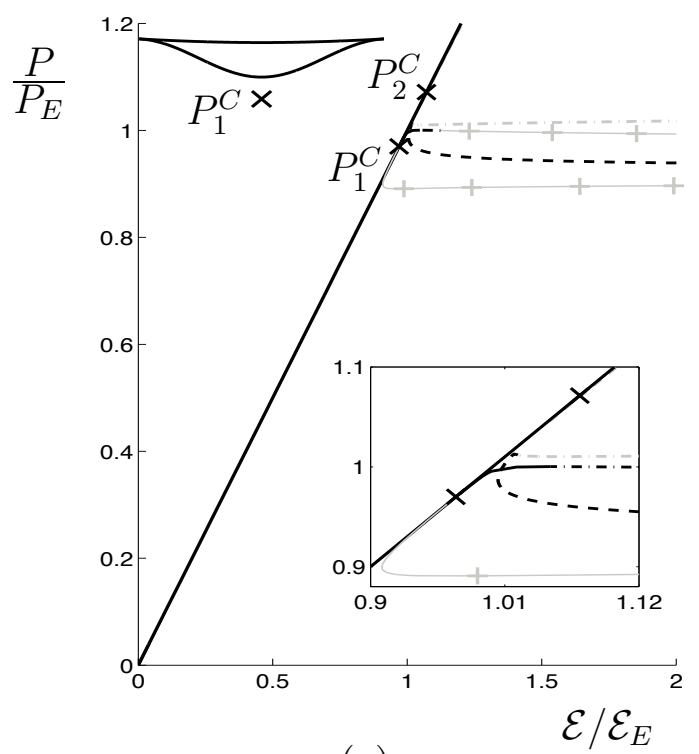

(a)

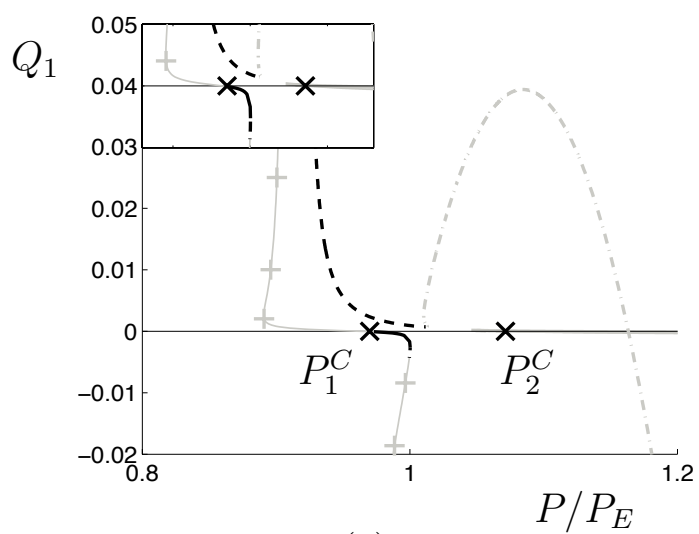

(c)

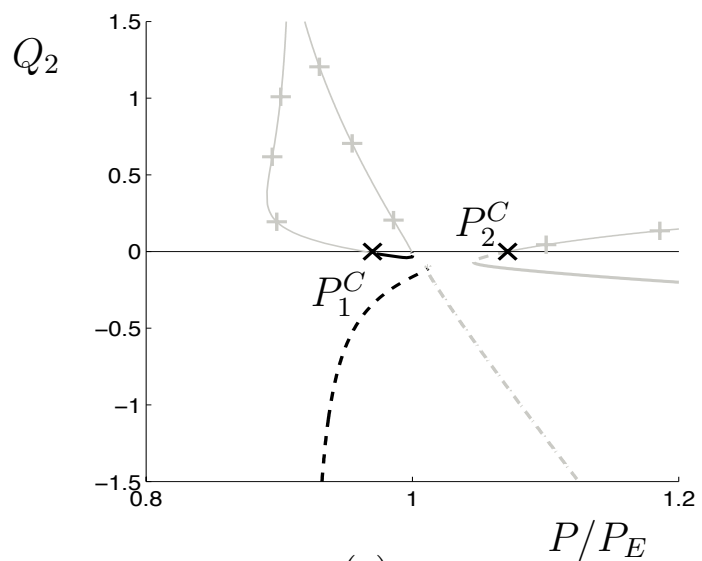

(e)

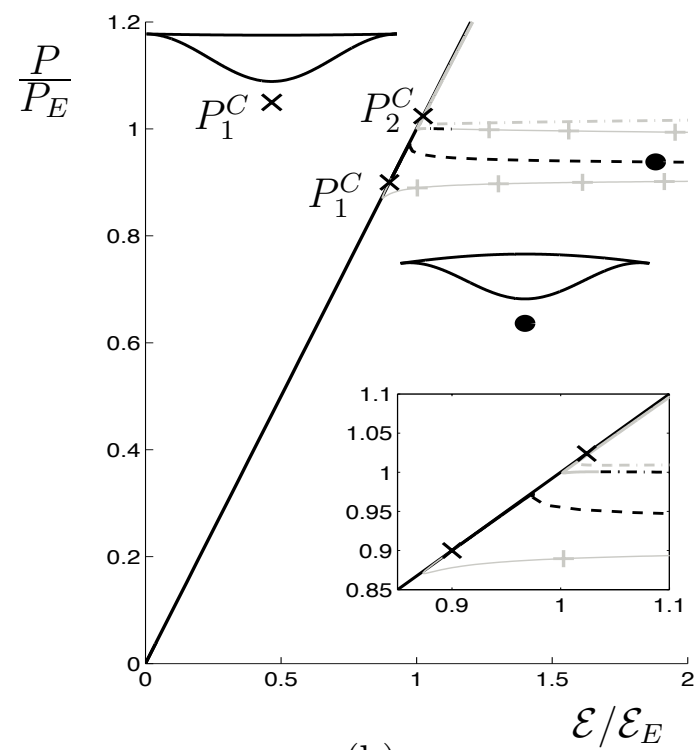

(b)

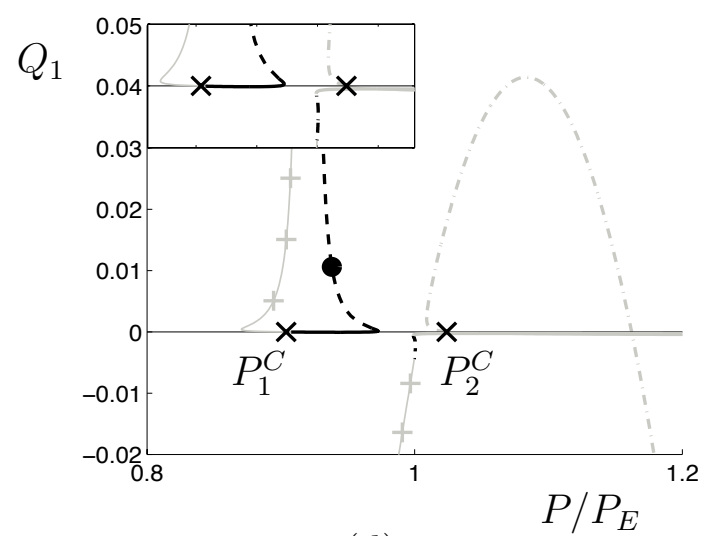

(d)

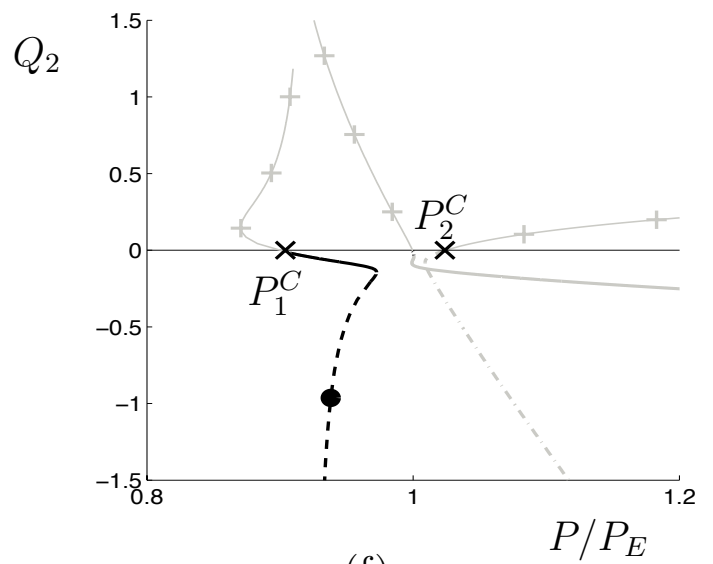

(f)

Figure 11: Load-end-shortening plots for $S=0.5, E_{1} / E_{2}=10$, (a) $a=0.79$, (b) $a=0.8$. Figures (c) - (f): The variation of the deflection with load. For clarity, the non-physical path emerging from $P_{2}^{C}$ is not crossed in Figures (a) - (d). 


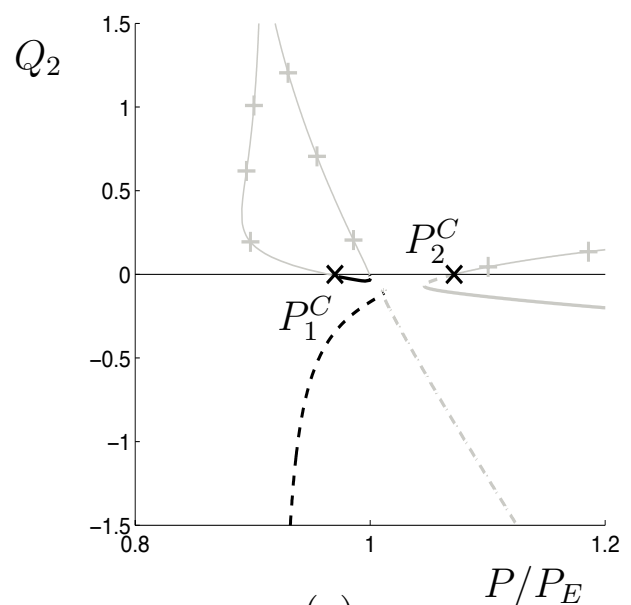

(a)

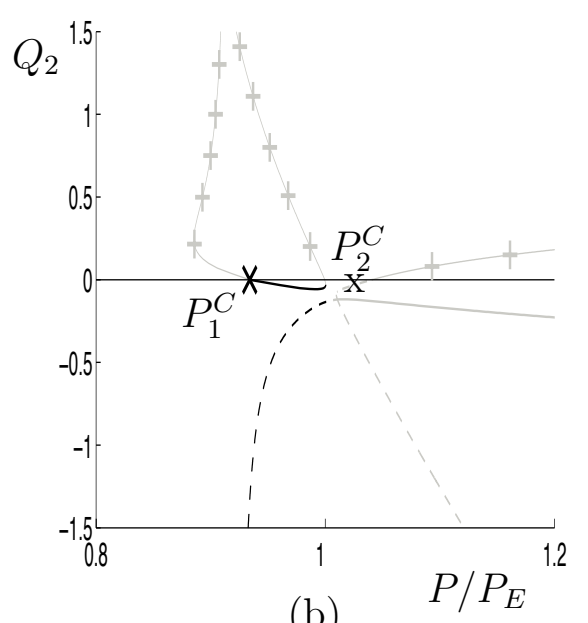

(b)

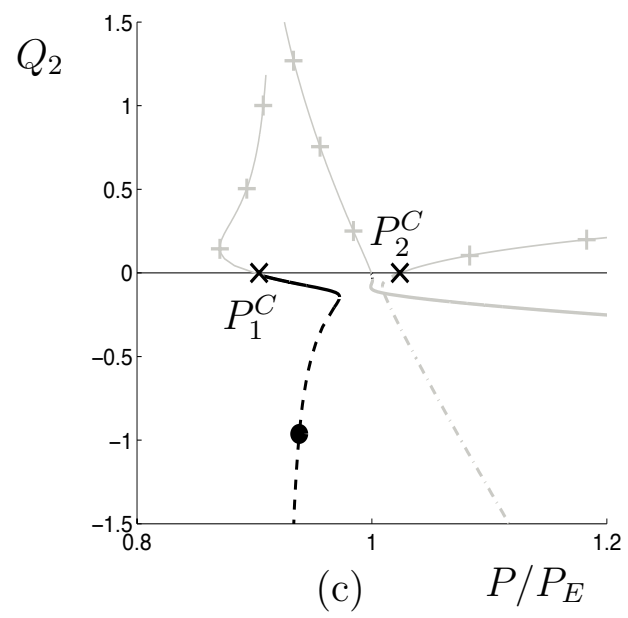

Figure 12: Two distinct bifurcations close together (a) $a=0.79$, (b) $a=0.795$, (c) $a=0.8$ 
A normal form for such a bifurcation, which is generally associated with a double eigenvalue is presented in (author?) (10, pgs 261-262) where three paths and the fundamental path meet at a bifurcation point. This is expounded further by (author?) (6) and is described in catastrophe theory terms using the umbilics (see also section ??). Further references may be found in (author?) (11) and (author?) (12) and a description is provided in chapter ??.

Examination of the plots in Figure 12 shows that the two secondary bifurcations also occur very close to the initial bifurcations from the fundamental path at the two critical buckling loads. In order for the phenomenon exhibited in the bimaterial strut to be accurately described as an elliptic umbilic it is required for there to be a point described as the umbilic point, ie a point at which all these four paths meet in a "stellar" bifurcation, when viewed on the three-dimensional $P-Q_{i}$ plots for $i=1,2$. Therefore the fundamental path is required as one of the bifurcation routes and the critical buckling loads and the analysis of chapter ?? and section ?? of this chapter are crucial to understanding this behaviour. The

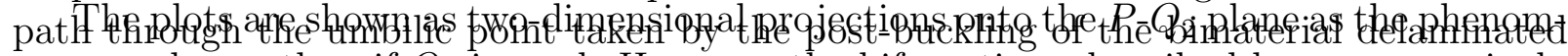
stratare gleafer than if $Q_{8}$ is used. However, the bifurcations described here are genuinely three-dimensional, occurring in all degrees-of-freedom or dimensions of the system.

In overview, Figure 13 shows how the limit points in $P$, labelled as $F_{i}$, approach, coincide and diverge. The fundamental path has been removed for clarity. If all these points $F_{i}$ and the two primary bifurcations from the fundamental path coincide at a point in parameter space then the umbilic point is achieved.

Plot (1) Two limit point $F_{1}$ and $F_{2}$ and a third path without folds in $P$.

Plot (2) A cusp arises on the third path as two folds $F_{3}$ and $F_{4}$ coincide. Two limit points $F_{1}$ and $F_{2}$ are also observed.

Plot (3) The cusp of Plot (2) has separated into two limit points $F_{3}$ and $F_{4}$. Two limit points $F_{1}$ and $F_{2}$ are also observed.

Plot (4) A transcritical bifurcation resulting from the coincidence of the $F_{2}$ and $F_{4}$ limit points. This annihilates $F_{2}$ and $F_{4}$. Two limit points $F_{1}$ and $F_{3}$ are also observed.

Plot (5) A depression is observed as the two paths involved in the transcritical bifurcation of Plot (4) unfold. Two limit points $F_{1}$ and $F_{3}$ are also observed.

Plot (6) Two limit points $F_{1}$ and $F_{3}$ are observed.

Plot (7) A transcritical bifurcation brings $F_{5}$ and $F_{6}$ into existence. Two limit points $F_{1}$ and $F_{3}$ are also observed.

Plot (8) Two limit points $F_{1}$ and $F_{3}$ are observed. Two further limitpoints $F_{5}$ and $F_{6}$ unfold the transcritical bifurcation of Plot (7).

Plot (9) A cusp occurs as $F_{1}$ and $F_{6}$ coincide. Two limit points $F_{3}$ and $F_{5}$ are also observed.

Plot (10) $F_{1}$ and $F_{6}$ have been annihiliated by the cusp. Two limit points $F_{3}$ and $F_{5}$ are also observed.

The crucial factors are the cusps of Plots (2) and (9). The umbilic point can only be found if these coincide. This sequence of events is significant evidence for the presence of the double tilted double cusp. It is the same as the sequence given by slicing through the elliptic umbilic except for the absence of the top and bottom edges (see section ?? and 4.14). These are provided by the critical load paths. 

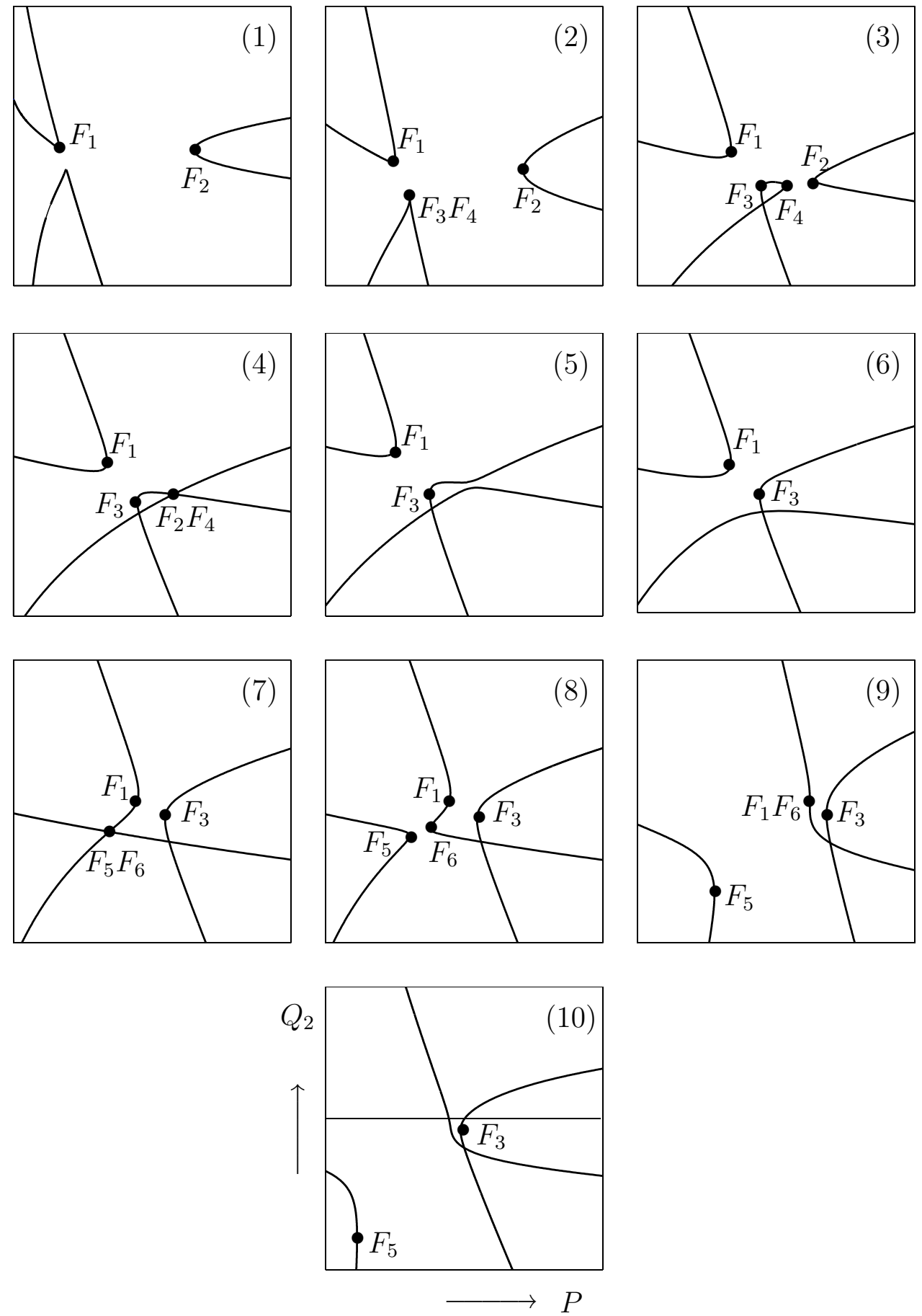

Figure 13: The cusp-fold collision sequence of the catastrophe exhibited by the delaminated bimaterial strut. The figures are plots with $Q_{2}$ on the vertical axes and normalized load $P$ on the horizontal axes. From left to right, working down the page, (1) $a=0.77$, (2) $a=0.786$, (3) $a=0.7913$, (4) $a=0.791451$, (5) $a=0.7915$, (6) $a=0.795$, (7) $a=0.79839$, (8) $a=0.7985,(9) a=0.803$, (10) $a=0.81$. The fundamental path is only shown in figure $(10)$. 


\subsection{The bifurcation set}

The bifurcation set of Figure 14 shows a profile similar to that of a slice through an asymmetric elliptic umbilic. This plots the set of limit points in terms of $P-a$ and for fixed $S=0.5$. The folds are shown separately with the external solid curves representing the points of initial bifurcation from the fundamental path (critical loads).

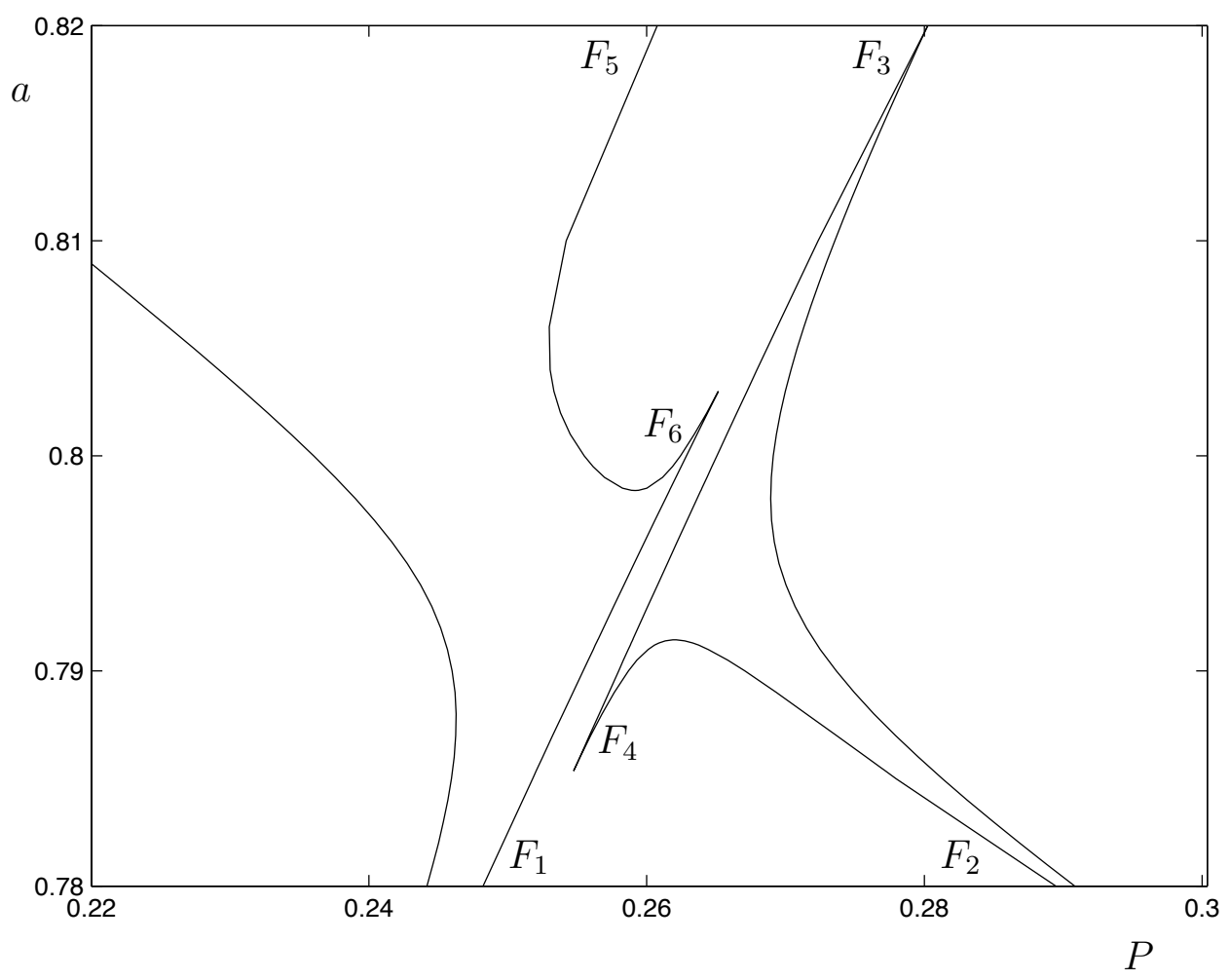

Figure 14: The bifurcation set for fixed $S=0.5$ : the slice of the catastrophe manifold

Notice that the curve for $F_{5}$ does not tend towards the critical curve or bottom edge of theelliptic umbilic as the $F_{2}$ curve does to the top edge. Instead, it unexpectedly veers away. This suggests a distortion in the surface of some kind and that the surface here is fully asymmetric. In fact, the structure being sliced is a double tilted double cusp which has elliptic and hyperbolic umbilics in its substructure.

\subsection{The potential surface}

Thus far, only slices through the surface have been considered. Instead, now consider the potential surface shown in Figure 15 as a contour plot in coordinate space. The aim is that the load value for which the plot is produced will be close to the load for the umbilic point. The plot is for a fixed value of $P=0.25$ and $a=0.794$. In order to calculate the value of $V$ at a point it was first necessary to calculate the corresponding value of $Q_{3}$. However, the computations failed above a particular value of $Q_{2}$ due to constraints of the numerical solvers in MatLab. It is still possible to see the important regions in both Figure 15 and Figure 16. The crosses mark the points at which the equilibrium paths intersect the surface for the chosen values of $P$ and $a$. 


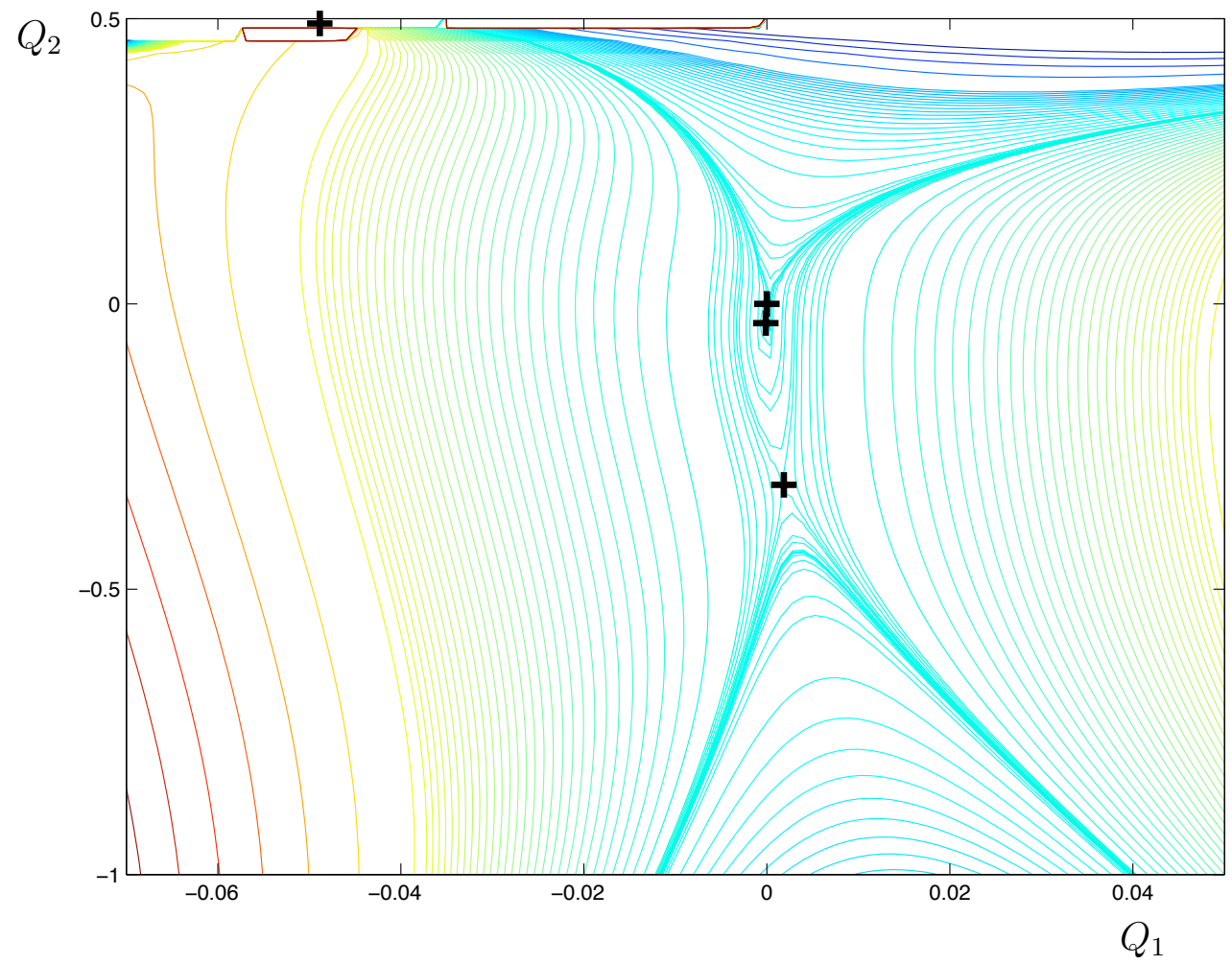

Figure 15: The potential surface for fixed $a=0.794, S=0.5, P=0.25$

Figure 16 is an enlarged view of the central section of the full potential surface shown in Figure 15. The crosses show that the equilibrium paths emerge from the potential surface mostly at points very close to one another, and, in particular, there are three stationary points together. This suggests the presence of an umbilic in the substructure of the catastrophe as per the analysis of (author?) (6) in which the surfaces with three converging roots are described as elliptic umbilic and hyperbolic umbilic while the hyperbolic umbilic also has a form with only one root. Clearly this umbilic is highly distorted in some way and the presence of other roots confirms that it is contained within some more complicated higher order structure. This more complicated structure could be well described using the terminology of the double cusp with some additional tilting parameters.

\subsection{Resulting physical behaviour}

The physical response of the structure is highly complex, especially in the region of the umbilic point. It is sensitive to small changes and perturbations, particularly in $a$ and $S$. Buckling could jump readily from one mode to another, possibly more dangerous, mode. The resulting behaviour is extremely unpredictable but is well described by a distorted umbilic within the substructure of a double tilted double cusp.

\subsection{Implications for an engineering structure}

On detecting a delamination in a critical area of an aircraft, the proximity of secondary bifurcations to the initial instability makes it very difficult to predict the effect of the delamination on the buckling response, particularly when using FEA. The double tilted 


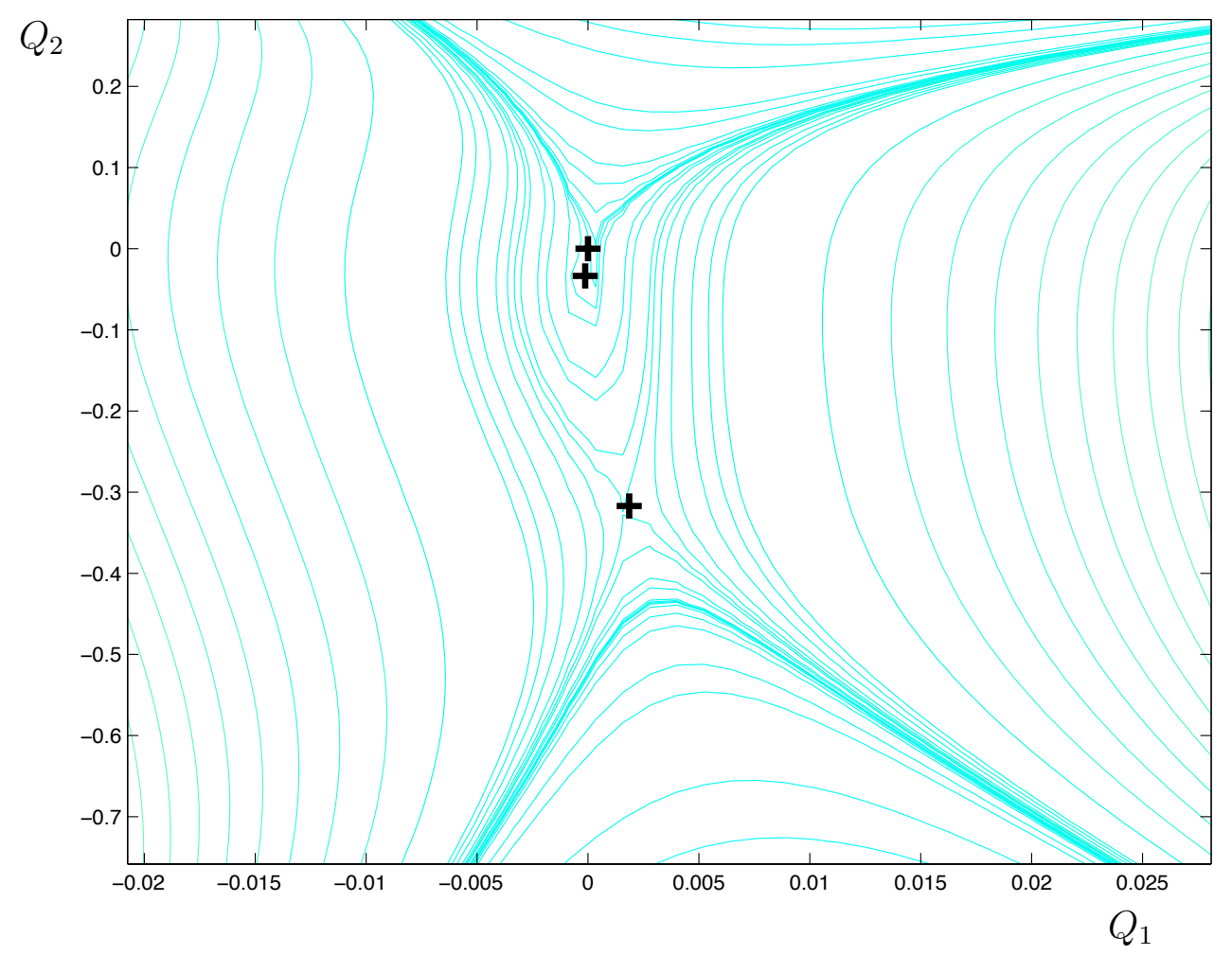

Figure 16: The central section of the potential surface for fixed $a=0.794, S=0.5$, $P=0.25$ 
double cusp is an extremelycomplicated object. The tilted cusp of chapter 5 (Figure ??) caused the response of the system to be highly unpredictable in the vicinity of the cusp. For the double tilted double cusp exhibited by the bimaterial strut this effect is potentially much worse. There are more possible routes that the post-buckling response may follow and predicting whether the safe closing or the dangerous opening reponse is seen is likely to be unreliable. Each case must be evaluated individually and accurately within the post-buckling range in order to be confident of its behaviour. However, the further the parameter set is from the umbilic point the more reliable the prediction of behaviour is. It is crucial that the effect of imperfections on the response is fully investigated alongside damage detection.

\section{Concluding Remarks}

In a move towards a model of the full composite material the bimaterial strut was introduced, comprising two layers of different depths and stiffnesses. A model in terms of a potential function was derived. Critical buckling loads and mode-shapes were obtained using a linear eigenvalue analysis and contrasted with those of the isotropic strut.

The post-buckling behaviour was examined and a fully asymmetric version of the elliptic umbilic catastrophe was found to be central to understanding the bifurcational response of the system. Bifurcation and catastrophe theory were used to completely explain the nature of the buckling response with the methods in (author?) (11) and (author?) (12).

The findings are summarised thus:

- A model describing the bimaterial strut in terms of total potential energy was presented. The equations were relatively simple to derive and the model easy to implement based on the implementation for the isotropic strut. However, the method presented here is much faster than FEA and is able to follow the solution through bifurcations.

- The "opening" and "closing" modes were found to be the initial buckling modes. Again, the "closing" mode is always observed for the lowest critical load. For $R=10$ and $S=0.5$ the modes are seen to draw close together around $a=0.25$ and $a=0.79$ between which the delamination is of little importance and neutral Euler buckling is observed. Further investigation of these regions enable us to find critical delamination depths For $a<0.25$ and $a>0.79$ the response is dominated by the buckling of the thinner laminate.

- The initial buckling response of the bimaterial strut contrasts with that of the isotropic strut as it is fully asymmetric. This asymmetry forces the two lower critical loads together and suggests the existence of a double eigenvalue. If this double eigenvalue exists then the two critical loads coincide and the initial response is highly unpredictable.

- The thin-film and overall buckling modes of chapter ?? are observed here with a critical delamination depth $a \approx 0.205$. The transition again takes place at a secondary bifurcation. Differences are observed in the response of $Q_{2}$ in thin-film buckling, causing a double reversal.

- The buckling patterns are more pronounced when $a>0.5, R>1$, equivalently, when the thicker layer is the stiffer of the two materials. 
- Two secondary bifurcations and two primary bifurcations can occur very close together in $\left(Q_{1}, Q_{2}, \cdots ; P, a, S, \cdots\right)$ space indicating the presence of an umbilic point and varying the parameters can move the bifurcations either closer together or further apart.

- The path through the double tilted double cusp as in this system is given and a slice through the distorted umbilic exhibited here shows how bringing $P_{1}^{C}$ and $P_{2}^{C}$ together moves the cusps and folds into coincidence yielding a double eigenvalue and the umbilic point.

- The bifurcation sets for various values of $S$ correspond to slices through a heavily distorted and fully asymmetric elliptic umbilic.

- Plots of the surface show the complexity and unpredictability of the post-buckling response of a compressed delaminated composite strut.

\section{References}

[1] G. W. Hunt. Buckling in space and time. To be published, 2005.

[2] G. W. Hunt, B. Hu, R. Butler, D. P. Almond, and J. E. Wright. Nonlinear modeling of delaminated struts. AIAA Journal, 42(11):2364-2372, 2004.

[3] C. J. Budd, R. Edmunds, and G. W. Hunt. A nonlinear model for parallel folding with friction. Proceedings of the Royal Society of London A. Mathematical, Physical and Engineering Sciences, 459(2036):2097-2119, 2003.

[4] S. P. Timoshenko and J. M. Gere. Theory of Elastic Stability. McGraw-Hill Book Company, second edition, 1961.

[5] J. M. T. Thompson and G. W. Hunt. A General Theory of Elastic Stability. John Wiley and Sons Ltd, 1973.

[6] J. M. T. Thompson and G. W. Hunt. Elastic Instability Phenomena. John Wiley and Sons Ltd, 1984.

[7] M. Golubitsky and D. G. Schaeffer. Singularities and Groups in Bifurcation Theory, volume 1 of Applied Mathematical Sciences 51. Springer-Verlag, 1985.

[8] M. J. D. Powell. A hybrid method for nonlinear equations. In P. Rabinowitz, editor, Numerical Methods for Nonlinear Algebraic Equations, pages 87-114. Gordon and Breach Science Publishers, 1970.

[9] J. E. Wright. Compound bifurcations in the buckling of a delaminated composite strut. Nonlinear Dynamics, Sp.Is, 2005. Accepted for publication.

[10] S.-N Chow and J. K. Hale. Methods of Bifurcation Theory. Springer-Verlag, 1982.

[11] E. C. Zeeman. Catastrophe Theory Selected Papers 1972 - 1977. Addison-Wesley, 1977.

[12] T. Poston and I. Stewart. Catastrophe Theory and its Applications. Pitman, London, 1978. 
[13] A. Balfour and D. H. Marwick. Programming in Standard Fortran 7\%. Heinemann, 1979.

[14] M. Golubitsky, I. Stewart, and D. G. Schaeffer. Singularities and Groups in Bifurcation Theory. Volume 2 of Applied Mathematical Sciences 69 (7), 1988.

[15] H. B. Keller. Numerical Methods for Two-Point Boundary-Value Problems. Dover Publications, dover edition, 1992.

[16] D. J. Higham and N. J. Higham. Matlab Guide. SIAM, 2000.

[17] W. J. Supple, editor. Structural instability, fundamentals of post-buckling behaviour of structures. IPC Science and Technology Press Ltd., 1973.

[18] H. Chai, C. D. Babcock, and W. G. Knauss. One dimensional modelling of failure in laminated plates by delamination buckling. International Journal of Solids and Structures, 17(11):1069-1083, 1981. 


\section{A Total Potential Energy Function}

The total potential energy function given below was obtained using the algebraic manipulation package Maple by the method described in section ?? (2).

$$
\begin{gathered}
V\left(Q_{1}, Q_{2}, Q_{3}, Q_{4} ; P\right)=\frac{1}{288 L^{3}}\left[9 E_{1} a t \pi^{4} Q_{1}^{4}+9 E_{2}(1-a) t \pi^{4} Q_{2}^{4}\right. \\
+4 \alpha t L Q_{3}^{4}+72 E_{1} a t \pi^{2} L Q_{1}^{3} Q_{3}+72 E_{2}(1-a) t \pi^{2} L Q_{2}^{3} Q_{3} \\
+48 E_{1} a t L^{3} Q_{1} Q_{3}^{3}+48 E_{2}(1-a) t L^{3} Q_{2} Q_{3}^{3}+12\left(12+\pi^{2}\right) E_{1} a t L^{2} Q_{1}^{2} Q_{3}^{2} \\
+12\left(12+\pi^{2}\right) E_{2}(1-a) t L^{2} Q_{2}^{2} Q_{3}^{2}+72 \frac{E_{1} E_{2} a(1-a)}{\alpha} t^{2} \pi^{2} L Q_{1}^{2} Q_{3} \\
-72 E_{1} a t \pi^{2} L Q_{1}^{2} Q_{4}-72 \frac{E_{1} E_{2} a(1-a)}{\alpha} t^{2} \pi^{2} L Q_{2}^{2} Q_{3} \\
-72 E_{2}(1-a) t \pi^{2} L Q_{2}^{2} Q_{4}+288 E_{1} E_{2} a(1-a) t^{2} L^{2} Q_{1} Q_{3}^{2} \\
-288 E_{1} E_{2} a(1-a) t^{2} L^{2} Q_{2} Q_{3}^{2}-48 \alpha t L^{3} Q_{3}^{2} Q_{4}-288 E_{1} a t L^{2} Q_{1} Q_{3} Q_{4} \\
-288 E_{2}(1-a) t L^{2} Q_{2} Q_{3} Q_{4}+24 E_{1} a^{3} t^{3} \pi^{4} Q_{1}^{2}+24 E_{2}(1-a)^{3} t^{3} \pi^{4} Q_{2}^{2} \\
+12 \frac{\gamma}{\alpha} t^{3} L^{2}\left(\beta^{2} S L^{2} \sec ^{2}(\beta S L)-\beta L \tan (\beta S L)+4\right) Q_{3}^{2}+144 \alpha t L^{2} Q_{4}^{2} \\
\left.-144 P L^{3}\left(Q_{3}^{2}\left(S \sec ^{2}(\beta S L)+\frac{1}{\beta} \tan (\beta S L)\right)+2 Q_{4}\right)\right]
\end{gathered}
$$

where

$$
\begin{aligned}
\alpha & =E_{1} a+E_{2}(1-a) \\
\beta & =\sqrt{\frac{P}{(E I)_{3}}} \\
\gamma & =E_{1}\left(E_{1}-E_{2}\right) a^{4}-E_{2}\left(E_{1}-E_{2}\right)(1-a)^{4}+E_{1} E_{2}
\end{aligned}
$$

